\title{
Identification of Electrical Faults in Underground Cables Using Machine Learning Algorithm ${ }^{+}$
}

\author{
Paramasivam Alagumariappan ${ }^{1, *}$, Mohamed Shuaib Y $^{1}$, Sonya A $^{2}$ and Irum Fathima ${ }^{1}$ \\ 1 Department of Electrical and Electronics Engineering, B. S. Abdur Rahman Crescent Institute of Science \\ and Technology, Chennai 600048, India; mdshuaiby@gmail.com (M.S.); \\ psinnovativeprojects@gmail.com (I.F.) \\ 2 Department of Information Technology, B. S. Abdur Rahman Crescent Institute of Science and Technology, \\ Chennai 600048, India; sonya.yasmin152@gmail.com \\ * Correspondence: parama.ice@gmail.com; Tel.: +91-984-378-0801 \\ + Presented at the 6th International Electronic Conference on Sensors and Applications, \\ 15-30 November 2019; Available online: https://ecsa-6.sciforum.net/.
}

Published: 14 November 2019

\begin{abstract}
Transmission and distribution play a vital role in delivering electricity. The presence of any fault in these systems may stop the delivery of electricity, which may create a huge problem in today's world. Hence, fault detection has become essential for delivering uninterrupted power supply. In this work, a portable and intelligent system is designed, and the fault detection on underground transmission lines is done using a developed hardware system. Also, the proposed system has a thermal camera which is an $8 \times 8$ array of infrared thermal sensors interfaced with a system-on-chip device, which collects the real-time thermal images when connected to the device. Further, the thermal camera returns an array of 64 individual infrared temperature readings of the transmission line and locates the point of damage that might occur due to the aging of conductor insulation, physical force, etc. Also, 200 images with thermal information from the different instances and directions are utilized to train the adapted machine learning algorithm. The python software is utilized to code the machine learning algorithm inside the system-on-chip device. The convolutional neural network-based machine learning algorithm is adopted and validated using various performance metrics such as accuracy, sensitivity, specificity, precision, negative predicted value, and F1_score. Results demonstrate that the proposed hardware is highly capable of locating faults in underground transmission lines.
\end{abstract}

Keywords: convolutional neural network; electrical faults; system on-chip; transmission line

\section{Introduction}

In recent years, electricity has become one of the survival factors. Electricity, being a form of energy that is converted from various other forms of energy, has to be transmitted from one place to another so that it is distributed to everyone. Energy exists in various forms. According to many researchers, the most commonly used form of energy is electrical energy, which is produced from various sources like coal, solar, nuclear etc. [1-5]. As it is possible to produce clean electrical energy using various renewable sources like wind mill, solar, hydro etc. and these are considered from generation point of view, the other two important aspects are transmission and power distribution. Transmission is better if produced energy is in the form of an alternating current (AC) instead of a direct current as it is easier and more cost effective to step-up or step-down the voltage level with minimal losses. 
Transmission and distribution play a vital role in delivering electricity. The presence of any faults in these systems may stop the delivery of electricity, which is a huge problem in today's life. Hence, fault detection has become essential for delivering uninterrupted power supply. Faults occur due to various reasons and can be classified either as man-made or a natural disaster. Faults that are manmade are more likely to be more predictable when compared to the faults occurring due to natural disasters, as it cannot be predicted when, where and how it will happen. Bulent and Donmez [6] (2010) have proposed that natural disasters not only fault the power system but also create disturbances in the power system and tend to cause blackouts. Musa et al. [7] (2018) developed a technique for the detection of faults in power transmission systems, focusing on the fusion of the current signal co-variance with the cumulative approach during a power swing. Further, the authors have tested for different fault conditions and the results showed a reasonable time response.

Ashrafi et al. [8] (2015) proposed a method for identifying DC faults in a transmission system with the help of a VSC (voltage source converter) HVDC system using sheath voltage, transient voltage and wavelet transform to distinguish the different DC faults. Further, the authors have concluded that the proposed method is a fast, reliable and novel strategy for detection of DC faults. Samantaray [9] (2009) discussed the identification of a fault zone in a flexible AC transmission line with the help of a decision tree (DT). Further, the authors have experimented with the fault inspection by considering one cycle of current and voltage samples post fault as an input vector which is given against the target output ' 1 ' for faults succeeding the flexible AC equipment's in line, and the target output is ' 0 ' for faults proceeding the flexible AC equipment's in line. Also, the authors have concluded that the outputs of this system were dependable for fault zone detection and categorization of fault in a flexible AC transmission line.

Many researchers have demonstrated the significance of Raspberry PI in various research works and projects irrespective of their fields, from agriculture to medicine. Raspberry PI has been an important part in various innovative researches like health care-based IOT, iris movement-based wheelchair control, robotic arm control in space with color recognition, automated blood bank, WSNbased automatic irrigation, and security systems [10-14]. Recently, thermal cameras have proved their strength through various innovations that are not only beneficial from the business point of view but also for the greater good of humanity, being used in the medical field for the detection of various types of disease like breast cancer, thyroid disease, Reynaud's phenomenon and so on. Thermal cameras have also been used in neonatal intensive care units to measure the body temperature as it is accurate and a non-contact type method and has also proven its worth in security fields as it is deployed for face recognition, proving to be far more secure than normal facial recognition systems [15-17].

Once the fault detection in transmission lines is a non-contact type, then the mechanism will have diverse advantages over the contact type fault detection. The advantages incorporate lesser operating time, minimizing the deterioration of quality material, reduced use of labor, and it can be deployed from any location as it can be connected using the cloud. The objective of this work is to simplify the method of fault detection by designing a compact mobile non-contact type fault detector capable of capturing thermal images of conductors and determining the location of faults.

\section{Materials and Methods}

\subsection{Thermal Image Acquisition System}

Figure 1 shows the block diagram of the proposed system for the identification of electrical faults. Further, the proposed system consists of a thermal camera and Raspberry PI-based systemon-chip device. In this work, the thermal camera AMG8833 consisting of an $8 \times 8$ array of IR thermal sensors is utilized for the acquisition of thermal images. Also, the AMG8833 type thermal camera module is connected directly to the Raspberry PI-based system-on-chip device using Inter-Integrated Circuit (I2C) protocol. The AMG8833 type thermal camera module captures thermal images of the conductors, which are given as inputs to the Raspberry PI, where it converts the given thermal image 
into a corresponding matrix and stores it. Further, these stored image matrices are utilized to train the adopted Convolutional Neural Network (CNN) for the classification process.

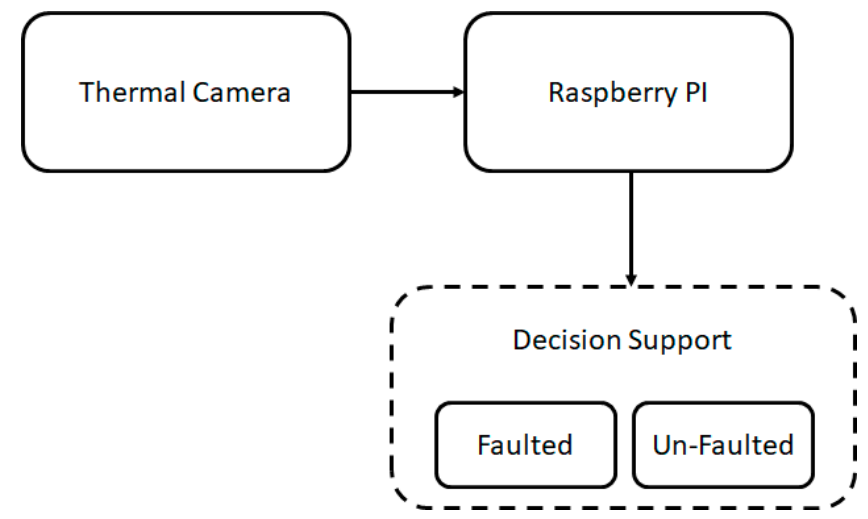

Figure 1. Block Diagram of a proposed system for the identification of electrical faults.

\subsection{Convolutional Neural Network}

In this work, the deep learning model is developed using Convolutional Neural Networks with LetNet architecture. The LeNet architecture consists of two sets of convolutional, activation, and pooling layers, followed by a fully-connected layer, activation, another fully-connected, and finally a softmax classifier $[18,19]$. The CNN classifier with LeNet network architecture has been implemented using Keras and Python. Also, a total of 200 images are utilized to train the CNN classifier, and an additional 100 images are utilized for testing the performance of the classifier. In short, the adopted CNN architecture can be represented as [INPUT-CONV-RELU-POOL-FC], which is explained as:

- INPUT [32 $\times 32 \times 3$ ] holds the raw pixel values of the image, whereas the image has a width of 32 , height of 32, and three color channels, R, G, B.

- CONV layer computes the output of the neurons that are connected to local regions in the input, each computing a dot product between their weights and a small region they are connected to in the input volume. This may result in a volume such as [32 $\times 32 \times 42]$ if the filter size is set to 42 .

- $\quad$ RELU layer applies an elementwise activation function, such as the $\max (0, x)$ thresholding at zero. This leaves the size of the volume unchanged $([32 \times 32 \times 42])$.

- POOL layer performs a down-sampling operation along the spatial dimensions (width, height), resulting in volume such as $[16 \times 16 \times 42]$.

- FC or Fully-Connected layer computes the class scores, resulting in the volume of size $[1 \times 1 \times$ 2], where each of the two numbers correspond to a class score, such as among the two categories (Faulted or Un-faulted).

\subsection{Performance Metrics}

A total of six metrics such as accuracy, sensitivity, specificity, Positive Predictive Value (PPV), Negative Predictive Value (NPV), and F1 score are computed to evaluate the performance of two different classification systems. The following equations are used to compute the performance metrics of the adopted CNN-based classification system.

$$
\begin{gathered}
\text { Accuracy }=(T P+T N) /(T P+F P+T N+F N) \\
\text { Sensitivity }=T P /(T P+F N) \\
\text { Specificity }=T N /(T N+F P)
\end{gathered}
$$

$$
\text { Positive predictivevalue }=T P /(T P+F P)
$$




$$
\begin{gathered}
\text { Negative predictive value }=T N /(T N+F N) \\
F-\text { Measure }=2 *\left(\frac{\text { Precision } * \text { Recall }}{\text { Precision }+ \text { Recall }}\right)
\end{gathered}
$$

where, TP is True Positive, TN is True Negative, FP is False Positive, and FN is False Negative.

\section{Results and Discussion}

The proposed hardware was connected to the computer system for analysis purposes. A total of 200 thermal images taken at different instances and directions were utilized to train the adopted CNN classifier. Further, 100 thermal images were utilized as the test image to analyze the performance of the adopted CNN-based decision support system.

The 100 test images with un-faulted and faulted conductors are given to the CNN classifier. Figure 2a,b shows the output of the CNN-based decision support system namely un-faulted and faulted respectively. It is seen that the $\mathrm{CNN}$ classifier gives a confidence level of $99.90 \%$ for un-faulted the condition. Further, it is seen that the CNN classifier gives a confidence level of $99.53 \%$ for the faulted condition. Also, it is observed that there is a significant change while comparing thermal images acquired from both faulty and un-faulty conductors. This is due to the heat generated by the flow of the current through the conductor. The amount of current flowing through the conductor changes with respect to its geometry. However, the variation in thermal signatures cannot be visualized or identified normally; the adopted CNN-based decision support system classifies faulty and un-faulty conductors accurately.

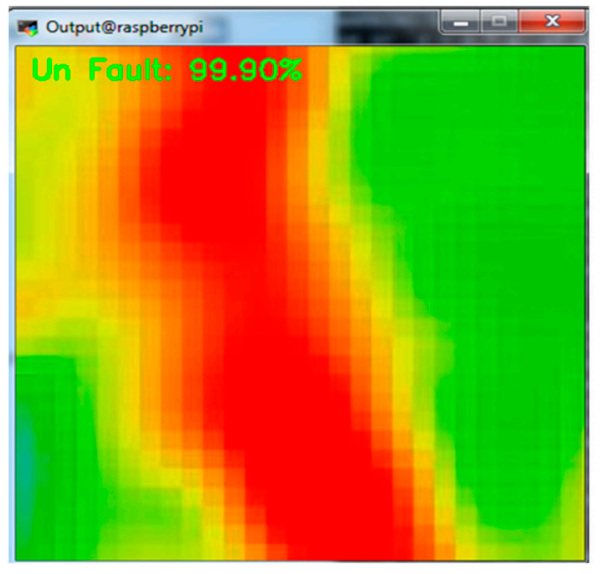

(a)

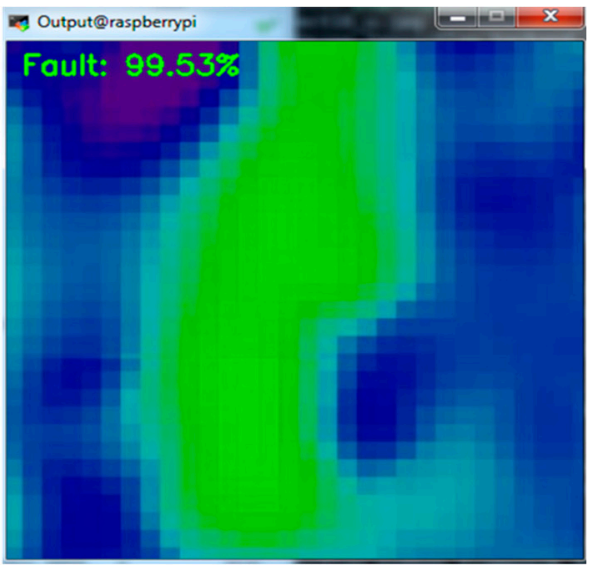

(b)

Figure 2. The output of a CNN-based decision support system on input thermal test imaged: (a) Un-faulty condition; (b) Faulty condition.

The performance measures such as accuracy, sensitivity, specificity, Positive Predictive Value (PPV), Negative Predictive Value (NPV), and F1 score of the CNN classifier are presented in Table 1. It is seen that the accuracy and sensitivity of the adopted CNN classifier are $93 \%$ and $91 \%$ respectively. Further, the specificity of the adopted CNN classifier is $95 \%$. Also, it is observed that the performance of a $\mathrm{CNN}$ classifier can be improved by increasing train images. 
Table 1. Performance Measures of Convolutional Neural Network Classifier.

\begin{tabular}{cc}
\hline Performance Measures & Convolutional Neural Network \\
\hline Accuracy (\%) & 93 \\
Sensitivty (\%) & 91 \\
Specificity (\%) & 95 \\
PPV (\%) & 95 \\
NPV (\%) & 90 \\
F1_Score & 0.93 \\
\hline
\end{tabular}

\section{Conclusions}

In this work, a hardware system with an AMG8833 thermal camera module and Raspberry PI was developed for the acquisition and identification of faults in underground cables. Further, the Convolutional Neural Network (CNN)-based classifier was utilized for the automated decision support system. Also, the python programming language was used to code a CNN-based machine learning algorithm. Results demonstrate that the accuracy, sensitivity and specificity of the adopted CNN classifier are 93\%, 91\% and 95\%, respectively. Further, the PPV, NPV and F1_Score of the adopted CNN classifier are $95 \%, 90 \%$ and 0.93 , respectively. The output of the CNN classifier acts as a decision support to classify faulty and un-faulty conductors. This work appears to be highly capable of locating faults in underground transmission lines.

Author Contributions: P.A. and M.S.Y. conceived the idea of this manuscript. I.F. designed the experiments and acquired the thermal images. P.A. and S.A have performed the analysis. All the authors have contributed towards preparing the manuscript. All the authors have read and approved the final manuscript.

Conflicts of Interest: The authors declare no conflict of interest.

\section{References}

1. Orosco, E.V. Simulation results of an efficient and innovative sun engine for solar to electrical energy conversion. In Collection of Technical Papers, Proceedings of the 35th Intersociety Energy Conversion Engineering Conference and Exhibit (IECEC) (Cat. No. 00CH37022), Las Vegas, NV, USA, 24-28 July 2000; American Institute of Aeronautics and Astronautics: Reston, VA, USA, 2000; Volume 2, pp. 908-914.

2. Gurkaynak, Y.; Li, Z.; Khaligh, A. A novel grid-tied, solar powered residential home with plug-in hybrid electric vehicle (PHEV) loads. In Proceedings of the 2009 IEEE Vehicle Power and Propulsion Conference, Dearborn, MI, USA, 7-10 September 2009; pp. 813-816.

3. Lyons, J.P.; Michael, C.R.; Paul V.; Robert, W.T. Wind Turbine Technology - The Path to 20\% US Electrical Energy. In Proceedings of the 2008 IEEE Power and Energy Society General Meeting-Conversion and Delivery of Electrical Energy in the 21st Century, Pittsburgh, PA, USA, 20-24 July 2008; pp. 1-4.

4. Kumar, A.; Karandikar, P.B.; Datta, S. Chavan. Generating and saving energy by installing wind turbines along the railway tracks. In Proceedings of the 2015 International Conference on Energy Systems and Applications, Pune, India, 30 October-1 November 2015; pp. 25-27.

5. ElKhodary, S.M.; Mahmoud, H.M.; Qotb, S.A.; and Sharouda, E.E.D.F. The role of nuclear energy in the future of energy systems in Egypt. In Proceedings of the 2017 Nineteenth International Middle East Power Systems Conference (MEPCON), Cairo, Egypt, 19 December 2017; pp. 638-643.

6. Oral, B.; Ferdun, D. The Impacts of natural disasters on power systems: anatomy of the Marmara earthquake blackout. Bülent, and Ferdun Dönmez. Acta Polytechnica Hungarica 2010, 7, 107-118.

7. Musa, M.H.H.; He, Z.; Fu, L.; Yujia, D. A covariance indices based method for fault detection and classification in a power transmission system during power swing. Int. J. Electr. Power Energy Syst. 2019, 105, 581-591.

8. Niaki, S.H.; Ashrafi, H. Karegar, K.; Ghalei, M.M. A novel fault detection method for VSC-HVDC transmission system of offshore wind farm. Int. J. Electr. Power Energy Syst. 2015, 73, 475-483.

9. Samantaray, S.R. Decision tree-based fault zone identification and fault classification inflexible AC transmissions-based transmission line. IET Gener. Transm. Distrib. 2009, 3, 425-436. 
10. Gupta, M.; Deekshith, S.; Patchava, V.; Menezes, V. Healthcare based on iot using raspberry pi. In Proceedings of the 2015 International Conference on Green Computing and Internet of Things (ICGCIoT), Greater Noida, India, 8-10 October 2015; pp. 796-799.

11. Sharma, J.M.; Anbarasu, C.C.; Shanmugasundaram, M. Iris movement based wheel chair control using raspberry Pi-A state of art. In Proceedings of the 2017 Innovations in Power and Advanced Computing Technologies (i-PACT), Vellore, India, 21-22 April 2017; pp. 1-5.

12. Szabó, R.; Gontean, A.; Sfiraț, A. Robotic arm control in space with color recognition using a Raspberry PI. In Proceedings of the 2016 39th International Conference on Telecommunications and Signal Processing (TSP), Vienna, Austria, 27-29 June 2016; pp. 689-692.

13. Adsul, A.C.; Bhosale, V.K.; Autee, R.M. Automated blood bank system using Raspberry PI. In Proceedings of the 2018 2nd International Conference on Inventive Systems and Control (ICISC), Coimbatore, India, 19-20 January 2018; pp. 252-255.

14. Kumar, K.; Kishore, S.; Durai, M.; Vadivel, T.; Kumar, K.A. Smart traffic system using raspberry pi by applying dynamic color changer algorithm. In Proceedings of the 2017 IEEE International Conference on Smart Technologies and Management for Computing, Communication, Controls, Energy and Materials (ICSTM), Chennai, India, 2-4 August 2017; pp. 146-150.

15. Guzman, A.M.; Goryawala, M.; Wang, J.; Barreto, A.; Andrian, J.; Rishe, N.; Adjouadi, M. Thermal imaging as a biometrics approach to facial signature authentication. IEEE J. Biomed. Health Inform. 2012, 17, $214-222$.

16. Sruthi, S.; Sasikala, M. A low cost thermal imaging system for medical diagnostic applications. In Proceedings of the 2015 International Conference on Smart Technologies and Management for Computing, Communication, Controls, Energy and Materials (ICSTM), Chennai, India, 6-8 May 2015; pp. 621-623.

17. Savaşci, Duygu, and Murat Ceylan. Thermal image analysis for neonatal intensive care units (First evaluation results). In Proceedings of the 2018 26th Signal Processing and Communications Applications Conference (SIU), Cesme, Izmir, 2-5 May 2018; pp. 1-4.

18. Golovko, V.; Myroslav, K.; Anatoly, S. Principles of neural network artificial immune system design to detect attacks on computers. In Proceedings of the 2010 International Conference on Modern Problems of Radio Engineering, Telecommunications and Computer Science (TCSET), Lviv-Slavske, Ukraine, 23-27 February 2010; pp. 237-237.

19. Mazare, A.; Ionescu, L.-M.; Lita, A.-L.; Serban, G.; Ionut, M. Mobile system with real time route learning using Hardware Artificial Neural Network. In Proceedings of the 2015 7th International Conference on Electronics, Computers and Artificial Intelligence (ECAI), Bucharest, Romania, 25-27 June 2015; p. 45.

(C) 2019 by the authors. Licensee MDPI, Basel, Switzerland. This article is an open access article distributed under the terms and conditions of the Creative Commons Attribution (CC BY) license (http://creativecommons.org/licenses/by/4.0/). 